\title{
Optimal Reactive Power Dispatch for Voltage Stability Improvement using Enhanced Whale Optimization Algorithm
}

\section{Gayathri Devi K.S.}

Noorul Islam Center for Higher Education, Kumaracoil, Thuckalai, Tamil Nadu, India ksgayathridevi2@gmail.com

\begin{abstract}
In recent days, the ORPD topic has attained significant consideration, since it enhances the economy and security of a power system. It includes discrete and continuous controlling parameters and thus it is considered as a complicated nonlinear issue. Thereby, this paper focuses on foremost objectives such as, diminution of loss and variations in voltage, which assists in resolving the ORPD problem under unbalanced states. For attaining these objectives, "Whale Optimization Algorithm based Parameter Optimization (WOAPO) approach" is exploited. This algorithm makes an impact on the controlling constraints that are adjusted to accomplish the optimal outcome. Finally, the superiority of the presented work is proved other other existing schemes. On observing the outcomes, the values attained under best case scenario using WOAPO scheme is $0.57 \%, 0.5 \%$ and $0.92 \%$ better than GA, FF and ABC. In addition, the worst case scenario of WOAPO approach is $1.16 \%$ superior to PSO model.
\end{abstract}

Keywords: ORPD; Voltage Profile; Power loss; Stability; VAR sources; WOAPO; Reactive power.

\begin{tabular}{ll} 
Nomenclature & \\
\hline Abbreviations & Descriptions \\
\hline APL & Active Power Loss \\
ABC & Artificial Bee Colony \\
2ArchMGWO & Two-Archive Multi-Objective Grey wolf Optimization \\
FF & FireFly \\
GA & Genetic Algorithm \\
MFO & Moth-Flame Optimization \\
NGBWCA & Gaussian bare-bones Water Cycle Algorithm \\
ORPD & Optimal Reactive Power Dispatch \\
OPF & Optimal Power Flow \\
PSO & Particle Swarm Optimization \\
RP & Reactive Power \\
WOAPO & Whale Optimization Algorithm based Parameter Optimization \\
\hline
\end{tabular}

\section{Introduction}

ORPD is a major aspect for OPF in modernized power systems for stable and secure operation. The most important task of such ORPD issue is to discover optimal settings of the entire controlling factors such as "reactive power output of generators, on-load tap changer of transformers, and shunt capacitor power outputs" [3]. In an electrical system, the ORPD is said to be a major factor for reducing losses and cost, for improving the stability of voltage and so on [1] [2].

The issue of reactive power dispatches for enhancing power system control and economical operations has attained much more consideration [4] [5] [6]. Moreover, a considerable sum of money could be saved by the decrease in active power loss accomplished from ORPD. The controlling parameters includes, "generator voltage magnitude, the tap ratios of transformer, static VAR sources" and so on. Accordingly, the parameters includes the "tap ratio limits, buses voltage limits, VAR source limits as well as voltage limits of generators" etc [11]. 
Several methods were introduced for the accomplishment of ORPD in power systems [7] [8] over the previous few decades. Traditional approaches such as "Mixed integer Programming, Predictor Corrector Primal Dual Interior Point Method, Nonlinear Programming, Linear Programming, gradient method, interior point method Successive Quadratic Programming" etc. are deployed for resolving ORPD issues [9] [10]. However, these approaches had failed to deal with the ORPD issues owing to their nonsmoothness and multi-modal characteristics.

The paper is arranged as: Section II portrays the reviews on ORPD and section III explains the modelling of ORPD under unbalanced conditions. Section IV elucidates the WOAPO based optimization for resolving ORPD issues. Finally, section V explains the results, and sections VI conclude the paper.

\section{Literature Review}

\subsection{Related works}

In 2014, Mojtaba et al. [1] established an effectual and consistent approach that depends upon ABC approach for resolving the ORPD issues. Accordingly, ABC approach is exploited for attaining "generator voltage magnitude, tap position of tap changing transformer and reactive power output of the compensation devices". Moreover, better convergence rate was attained that proved the superiority of presented model over the existing schemes.

In 2017, Ali et al. [2] introduced a NGBWCA oriented model for overcoming and dealing with the issues occurring in ORPD. In this work, the resistive losses and voltage discrepancies were considered as objectives that need to be reduced. The efficiency of the adopted NGBWCA method was examined over other schemes and the results were obtained. In the end, the numerical tests and experimental results has revealed the efficacy of the NGBWCA model in solving ORPD problems.

In 2017, Kasem et al. [3] established an original 2ArchMGWO approach for solving the issues in ORPD. In this context, the optimizer was improved from its usual form by the update of reproduction function and by summing the 2 -archive hypothesis to the system. In addition, the optimal outcomes obtained from a number of optimizers were evaluated over one another depending on hypervolume indicator, and it was proved that the adopted method was apparently enhanced than the compared ones.

In 2017, Rebecca et al. [4] introduced a new technique to cope up with the ORPD problem by adopting the concept of well known optimization scheme called MFO. In addition, optimal grouping of controlling parameters were carried out by means of MFO, by which minimal total power loss and minimal voltage deviations could be attained. Eventually, the enhancement of the presented approach was proved other existing schemes by carrying out mathematical analysis.

In 2015, Rajan and Malakar [5] had suggested a novel hybridized technique, which combined FF approach for resolving the issues associated with ORPD. In addition, it was observed that the adopted scheme has improved robustness and convergence rates when compared over the actual FF model and other conventional models. As a result, it was revealed that the introduced method has presented superior solutions.

\section{Modelling of ORPD Under Unbalanced Conditions}

\subsection{Objective Model}

The minimisation of APL and development of voltage stability is a most significant factor of ORPD. The dependent parameters is shown in Eq. (1), where $P_{G}$ symbolizes the dropped bus power, $V_{\text {li }}$ signifies the voltage bus PQ ( $\mathrm{i}=1,2 \ldots . \mathrm{MPQ}), \mathrm{Q}_{\mathrm{Gi}}$ indicates the $\mathrm{RP}$ output of generator $(\mathrm{i}=1,2 \ldots . \mathrm{MG}), \quad M G$ and MPQ refers to the count of generator bus and PQbus respectievly. The control variable vector is specified by Eq. (2).

$$
\begin{aligned}
& \mathrm{Z}=\left[\mathrm{P}_{\mathrm{G} 1}, \mathrm{~V}_{1-1} \ldots \ldots . \mathrm{V}_{1-\mathrm{MPQ}}, \mathrm{Q}_{\mathrm{G}-1} \ldots \ldots \mathrm{Q}_{\mathrm{G}-\mathrm{MG}}\right] \\
& \mathrm{J}=\left[\mathrm{V}_{\mathrm{G}-1}, \ldots . . \mathrm{V}_{\mathrm{G}-\mathrm{MG}}, \mathrm{Q}_{\mathrm{C}-1} \ldots . . \mathrm{Q}_{\mathrm{C}-\mathrm{MC}}, \mathrm{B}_{1}, \ldots . \mathrm{B}_{\mathrm{MB}}\right]
\end{aligned}
$$

In Eq. (2), " $\mathrm{V}_{\mathrm{Gi}}$ refers to the terminal bus voltage, $\mathrm{Q}_{\mathrm{Ci}}$ signifies the output of shunt VAR compensator, $\mathrm{B}_{\mathrm{i}}$ indicates the tap setting of tap changing transformer, $\mathrm{MC}$ and MBdenotes the count of shunt VAR compensator and tap clanging transformer" respectievly. 
The objective function of this work is specified in Eq. (3), in which $\mathrm{D}_{\mathrm{b}}$ point out APL, and $\mathrm{D}_{\mathrm{o}}$ signify the difference in voltage.

$$
f_{i}=\alpha D_{o}+(1-\alpha) D_{o}
$$

\subsection{Minimization of APL}

The APL minimisation is modelled as per Eq. (4), where $\mathrm{P}_{1}$ point out the APL, M refers to transmission line's count, $g_{k}$ points out the conductance of $\mathrm{k}^{\text {th }}$ branch. Also, $\delta_{\mathrm{n}}$ and $\delta_{\mathrm{m}}$ refers to the voltage phase angles of $\mathrm{n}^{\text {th }}$ and $\mathrm{m}^{\text {th }}$ buses in that order.

$$
\mathrm{D}_{\mathrm{b}}=\mathrm{P}_{1}=\sum_{\mathrm{k}=1}^{\mathrm{M}} \mathrm{g}_{\mathrm{k}}\left[\mathrm{V}_{\mathrm{m}}^{2}+\mathrm{V}_{\mathrm{n}}^{2}-2 \mathrm{~V}_{\mathrm{m}} \mathrm{V}_{\mathrm{n}} \cos \left(\delta_{\mathrm{m}}-\delta_{\mathrm{n}}\right)\right]
$$

\subsection{Voltage Variations}

The minimisation of voltage magnitude $\left(\mathrm{V}_{\mathrm{i}}\right)$ at diverse loads from a fixed reference value $\left(\mathrm{V}^{\text {ref }}\right)$ of $\mathrm{V}_{\mathrm{i}}$ is deployed for improving the voltage profile, which is specified in Eq. (5). Here, lb indicate the total count of load buses and $\psi(y)$ refers to the step function that is computed as per Eq. (6).

$$
\begin{aligned}
& D_{\mathrm{o}}=V_{\mathrm{F}}=\sum_{\mathrm{i}=1}^{\mathrm{lb}} \mathrm{P}_{\mathrm{f}} \psi\left(\mathrm{V}^{\mathrm{min}}-\mathrm{V}_{\mathrm{m}}\right)+\mathrm{P}_{\mathrm{f}} \psi\left(\mathrm{V}_{\mathrm{m}}-\mathrm{V}^{\mathrm{max}}\right) \\
& \psi(\mathrm{y})= \begin{cases}1 ; & \text { if } \mathrm{y} \geq 0 \\
0 ; & \text { otherwise }\end{cases}
\end{aligned}
$$

In Eq. (7), $\mathrm{Q}$ and $\mathrm{P}$ refers to the reactive and real powers, in that order. In addition, $y$ and $r$ signifies the susceptance and resistance of line respectively.

$$
\begin{aligned}
& \left|\mathrm{V}_{\mathrm{m}}\right|^{2}=\left|\mathrm{V}_{\mathrm{i}}\right|^{2}-2\left(\mathrm{P}_{\mathrm{im}} \tilde{\mathrm{r}_{\mathrm{im}}}+\tilde{\mathrm{y}}_{\mathrm{im}} \mathrm{Q}_{\mathrm{im}}\right)+\mathrm{c}_{\mathrm{im}}(\mathrm{P}, \mathrm{Q}) \\
& \tilde{r}_{i m}=\operatorname{Re}\left\{b b^{H}\right\} \otimes r_{i m}+\operatorname{Im}\left\{b b^{H}\right\} \otimes y_{i m} \\
& \tilde{\mathrm{y}}_{\mathrm{im}}=\operatorname{Re}\left\{b b^{\mathrm{H}}\right\} \otimes \mathrm{y}_{\mathrm{im}}-\operatorname{Im}\left\{b b^{\mathrm{H}}\right\} \otimes \mathrm{r}_{\mathrm{im}} \\
& b=\left[\begin{array}{lll}
1 & e^{-j 2 \pi / 3} & e^{j 2 \pi / 3}
\end{array}\right] \\
& \mathrm{c}_{\mathrm{im}}=\left[\mathrm{z}_{\mathrm{im}}\left[\mathrm{T}_{\mathrm{im}}^{*} \cdot / \mathrm{V}_{\mathrm{i}}^{*}\right]\right] \otimes\left[\mathrm{z}_{\mathrm{im}}^{*}\left[\mathrm{~T}_{\mathrm{im}_{0}} \cdot / \mathrm{V}_{\mathrm{i}}\right]\right] \\
& \mathrm{z}_{\mathrm{im}}=\mathrm{r}+\mathrm{jy} \\
& \mathrm{T}_{\mathrm{im}}=\left[\mathrm{P}_{\mathrm{im}}+\mathrm{jQ} \mathrm{Q}_{\mathrm{im}}\right] \otimes\left[\tilde{\mathrm{z}_{\mathrm{im}}}\left(\mathrm{P}_{\mathrm{im}}-\mathrm{jQ} \mathrm{Q}_{\mathrm{im}}\right)\right] \\
& \tilde{\mathrm{z}}_{\mathrm{im}}=\mathrm{z}_{\mathrm{im}} \otimes\left(\mathrm{b}_{\mathrm{i}} \overline{\mathrm{b}}_{\mathrm{i}}^{\mathrm{H}}\right)
\end{aligned}
$$

The $\mathrm{L}$-index value $\left(\mathrm{N}_{\mathrm{n}}\right)$ of $\mathrm{n}^{\text {th }}$ bus is specified as Eq. (15), where $\mathrm{n}=1,2 \ldots \ldots, \mathrm{MPQ}$, MPV signifies PV bus count and $X_{o}$ and $X_{b}$ of Eq. (16) refers to the sub-matrices. XBUS is specified by (17), after the partition of $\mathrm{PQ}$ and $\mathrm{PV}$ bus.

$$
\begin{aligned}
& \mathrm{N}_{\mathrm{n}}=\left|1-\sum_{\mathrm{m}=1}^{\mathrm{MPV}} \mathrm{D}_{\mathrm{nm}} \frac{\mathrm{V}_{\mathrm{m}}}{\mathrm{V}_{\mathrm{n}}}\right| \\
& \mathrm{D}_{\mathrm{nm}}=\left[\mathrm{X}_{\mathrm{b}}\right]^{-1}\left[\mathrm{X}_{\mathrm{o}}\right] \\
& {\left[\begin{array}{l}
\mathrm{I}_{\mathrm{PQ}} \\
\mathrm{I}_{\mathrm{PV}}
\end{array}\right]=\left[\begin{array}{l}
\mathrm{X}_{\mathrm{b}} \mathrm{X}_{\mathrm{o}} \\
\mathrm{X}_{\mathrm{c}} \mathrm{X}_{\mathrm{d}}
\end{array}\right]\left[\begin{array}{l}
\mathrm{V}_{\mathrm{PQ}} \\
\mathrm{V}_{\mathrm{PV}}
\end{array}\right]}
\end{aligned}
$$

The value of $\mathrm{N}_{\mathrm{n}}$ is fixed as 0 or 1 that depends on the voltage fall down and no load states of $\mathrm{n}^{\text {th }}$ bus. Thus, the objective function is specified by Eq. (18), in which $\mathrm{N}_{\mathrm{n}}=1,2 \ldots . . \mathrm{MPQ}$.

$$
\mathrm{D}_{\mathrm{c}}=\max \left(\mathrm{N}_{\mathrm{n}}\right)
$$




\subsection{Inequality and Equality Parameters}

The power system is regulated by equality factors and it involves the load flow of voltage, which are modelled as in Eq. (19) and Eq. (20).

$$
\begin{aligned}
& \mathrm{P}_{\mathrm{Gm}}-\mathrm{P}_{\mathrm{Dm}}-\mathrm{V}_{\mathrm{m}} \sum_{\mathrm{n}=1}^{\mathrm{MB}} \mathrm{V}_{\mathrm{n}}\left[\mathrm{F}_{\mathrm{mn}} \sin \left(\delta_{\mathrm{m}}-\delta_{\mathrm{n}}\right)+\mathrm{G}_{\mathrm{mn}} \cos \left(\delta_{\mathrm{m}}-\delta_{\mathrm{n}}\right)\right]=0 \\
& \mathrm{~m}=1,2 \ldots \ldots \ldots . . \mathrm{MB} \\
& \mathrm{Q}_{\mathrm{Gm}}-\mathrm{Q}_{\mathrm{Dm}}-\mathrm{V}_{\mathrm{m}} \sum_{\mathrm{n}=1}^{\mathrm{MB}} \mathrm{V}_{\mathrm{n}}\left[\mathrm{F}_{\mathrm{mn}} \sin \left(\delta_{\mathrm{m}}-\delta_{\mathrm{n}}\right)+\mathrm{G}_{\mathrm{mn}} \cos \left(\delta_{\mathrm{m}}-\delta_{\mathrm{n}}\right)\right]=0
\end{aligned}
$$

In Eq. (20), $\mathrm{MB}$ refers to bus count, $\mathrm{Q}_{\mathrm{Gm}}$ and $\mathrm{P}_{\mathrm{Gm}}$ refers to the generated reactive and active power at $\mathrm{m}^{\text {th }}$ bus, $\mathrm{Q}_{\mathrm{Dm}}$ and $\mathrm{P}_{\mathrm{Dm}}$ refers to demand associated with reactive and active powers, $B_{m n}$ and $G_{m n}$ signifies the transfer susceptance and conductance.

The upper and lower bounds for magnitude of RP and output voltage were specified by Eq. (21) and Eq. (22).

$$
\begin{aligned}
& \mathrm{Q}_{\mathrm{Gm}}^{\min } \leq \mathrm{Q}_{\mathrm{Gm}} \leq \mathrm{Q}_{\mathrm{Gm}}^{\max }, \mathrm{m}=1,2, \ldots \ldots . \mathrm{MG} \\
& \mathrm{V}_{\mathrm{Gm}}^{\min } \leq \mathrm{V}_{\mathrm{Gm}} \leq \mathrm{V}_{\mathrm{Gm}}^{\max } \mathrm{m}=1,2, \ldots \ldots . \mathrm{MG}
\end{aligned}
$$

The upper and lower bounds of RP at output in shunt VAR compensators are shown in Eq. (23).

$$
\mathrm{Q}_{\mathrm{Cm}}^{\mathrm{min}} \leq \mathrm{Q}_{\mathrm{Cm}} \leq \mathrm{Q}_{\mathrm{Cm}}^{\max } \mathrm{m}=1,2, \ldots \ldots . \mathrm{MC}
$$

In addition, the lower and upper values of transformer tap setting are shown by Eq. (24). A certain limit exists for the voltage in buses and all line flows are modelled as shown in Eq. (25) and Eq. (26).

$$
\begin{aligned}
& \mathrm{B}_{\mathrm{m}}^{\min } \leq \mathrm{B}_{\mathrm{m}} \leq \mathrm{B}_{\mathrm{m}}^{\max }, \mathrm{m}=1,2, \ldots \ldots . \mathrm{MB} \\
& \mathrm{V}_{\mathrm{Nm}}^{\min } \leq \mathrm{V}_{\mathrm{Nm}} \leq \mathrm{V}_{\mathrm{Nm}}^{\max } \mathrm{m}=1,2, \ldots \ldots . \mathrm{MPQ} \\
& \mathrm{T}_{\mathrm{lm}} \leq \mathrm{T}_{\mathrm{lm}}^{\max } \mathrm{m}=1,2, \ldots \ldots . \mathrm{M}
\end{aligned}
$$

\section{WOAPO based Optimization for Resolving ORPD Issues}

\subsection{Solution Encoding}

The parameters namely, transformer tap setting (B), voltage magnitude (V) and RP (Q) are provided as solutions to WOAPO approach for resolving the ORPD issues. The solution encoding is shown in Fig. 1. "The RP of 5 generator buses (bus no: 1, 2, 3, 6 and 8), voltage magnitude of bus 13 and 3, transformer tap setting of buses 8, 9 and 10" are fixed optimally via WOAPO approach, which minimizes the voltage divergence and power losses.

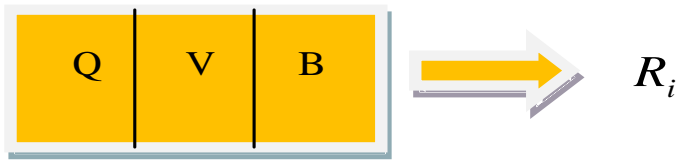

Fig. 1. Solution Encoding

\subsection{WOAPO Algorithm}

In the conventional WOA model, the main drawbacks are that it is not good at exploring the search space. Therefore, WOAPO is exploited in this work. The mathematical modelling of WOAPO [16] model is briefly explained here.

(i) Encircling Prey: The humpback whales have the capability to spot the locality of prey and encircle them. The encircling activities of humpback whales are specified in Eq. (27) and Eq. (28), where $\overrightarrow{\mathrm{A}}$ and $\overrightarrow{\mathrm{U}}$ refer to the coefficient vectors and current iteration is indicated as $\mathrm{t}$.

$$
\begin{aligned}
& \vec{H}=\left|\vec{U} \cdot \vec{R}_{p}(t)-\vec{R}(t)\right| \\
& \vec{R}(t+1)=\vec{R}_{p}(t)-\vec{A} \cdot \vec{H}
\end{aligned}
$$

In addition, $\vec{R}$ denotes the positions and $\vec{R}_{p}$ denotes best position acquired so far. Furthermore, $\overrightarrow{\mathrm{A}}$ and $\overrightarrow{\mathrm{H}}$ are computed as per Eq. (29) and Eq. (30). In Eq. (29), the component $\overrightarrow{\mathrm{a}}$ is lessened from 2 to 0 for diverse iterations. The random vectors $\mathrm{ra}_{1}$ and $\mathrm{ra}_{2}$ resides lies amongst $[0,1]$.

$$
\overrightarrow{\mathrm{A}}=2 \hat{\mathrm{a}} \cdot \mathrm{ra} \mathrm{a}_{1}-\overrightarrow{\mathrm{a}}
$$




$$
\overrightarrow{\mathrm{H}}=2 \mathrm{ra}_{2}
$$

(ii) Exploitation Phase:

It is modelled depending on the "Shrinking encircling mechanism and Spiral updating position". (a)"Shrinking encircling mechanism": It is accomplished by lessening the $\vec{a}$ value in Eq. (29).

(b) Spiral update Evaluation: A spiral formula is formed among the whale position and prey as represented in Eq. (31), in which $\overrightarrow{\mathrm{H}}$ indicates the distances of $\mathrm{i}^{\text {th }}$ whale to prey and $\mathrm{b}$ is a variable that denotes "logarithmic spiral shape" and $l$ is an arbitrary integer that lies between $[-1,1]$. The arithmetical formula for $\overrightarrow{\mathrm{H}}$ is given in Eq. (32).

$$
\begin{aligned}
& \mathrm{R}(\mathrm{t}+1)=\overrightarrow{\mathrm{H}}^{\prime} \mathrm{e}^{\mathrm{bl}} \cdot \operatorname{Cos}(2 \pi \mathrm{l})+\overrightarrow{\mathrm{R}}_{\mathrm{p}}(\mathrm{t}) \\
& \overrightarrow{\mathrm{H}}^{\prime}=\left|\overrightarrow{\mathrm{R}}_{\mathrm{p}}(\mathrm{t})-\overrightarrow{\mathrm{R}}(\mathrm{t})\right|
\end{aligned}
$$

During optimization, the position of whales gets updated, which is shown numerically in Eq. (33), in which $\phi$ refers to an arbitrary integer.

$$
R(t+1)=\left\{\begin{array}{ccc}
\vec{R}_{p}(t)-\vec{A} \cdot \vec{H} \quad \text { if } & \phi<0.5 \\
\vec{H}^{\prime} \cdot e^{b l} \cdot \operatorname{Cos}(2 \pi l)+\vec{R}_{p}(t) & \text { if } \quad \phi \geq 0.5
\end{array}\right.
$$

Exploration phase: This phase is evaluated as shown in Eq. (34) and Eq. (35). The arbitrary position vector elected from the present population is indicated by $R_{(\text {rand })}$.

$$
\begin{aligned}
& \overrightarrow{\mathrm{H}}=\left|\overrightarrow{\mathrm{A}} \overrightarrow{\mathrm{R}}_{(\text {rand })}-\overrightarrow{\mathrm{R}}\right| \\
& \overrightarrow{\mathrm{R}}(\mathrm{t}+1)=\overrightarrow{\mathrm{R}}_{(\text {rand })}-\overrightarrow{\mathrm{A}} \cdot \overrightarrow{\mathrm{H}}
\end{aligned}
$$

\section{Results and Discussions}

\subsection{Simulation Set up}

The accomplishment of ORPD using WOAPO approach was implemented in MATLAB, and the experimentation was carried out on "IEEE 14 and IEEE 39 bus systems". The analysis were performed for resolving ORPD issues under specific loading states such as voltage penalty $\left(F_{a}\right)$ and APL $\left(F_{b}\right)$. In addition, the superiority of WOAPO approach was evaluated over other existing optimization schemes such as, GA [12], FF [13], PSO [14] and ABC [15]. Moreover, statistical analysis was carried out for portraying the enhancement of WOAPO approach.

\subsection{Statistical Analysis}

The statistical analysis of WOAPO based approach for obtaining enhanced ORPD is specified by Table I and Table II for IEEE 14 and IEEE 19 bus systems. As the met heuristic models are stochastic, and to ensure fair comparison, each algorithm is executed ten times to obtain the statistics of the objective. On observing the outcomes, the values attained under best case scenario using WOAPO scheme is $0.57 \%$, $0.5 \%$ and $0.92 \%$ better than GA, FF and $\mathrm{ABC}$. In addition, the worst case scenario of WOAPO approach is $1.16 \%$ superior to PSO model. In addition, the values attained under mean case scenario using WOAPO technique is $0.12 \%, 0.12 \%, 0.78 \% \%$ better than GA, FF and ABC models. Likewise, from Table II, the WOAPO approach has attained a best case scenario that is $0.24 \%, 0.29 \%$ and $0.29 \%$ better than PSO, FF and GA models. Also, the mean case scenario of WOAPO scheme is $4.13 \%, 4.68 \%, 0.35 \%$ and 0.2 $\%$ better than GA, FF, PSO and ABC models. As a result, the improvement of WOAPO algorithm was established from the simulated outcomes.

Table 1: Statistical analysis: Evaluation of WOAPO approach over existing approaches on IEEE 14 bus system

\begin{tabular}{llllll}
\hline Metrics & FF [13] & GA [12] & ABC [15] & PSO [14] & WOAPO \\
\hline Best & 0.29496 & 0.29496 & 0.29496 & 0.296 & 0.29327 \\
Mean & 0.29496 & 0.29496 & 0.29496 & 0.29694 & 0.29462 \\
Standard deviation & $1.66 \times 10^{10}$ & $6.67 \times 10^{9}$ & $3.26 \times 10^{9}$ & 0.000933 & 0.000752 \\
Median & 0.29496 & 0.29496 & 0.29496 & 0.29693 & 0.29496 \\
Worst & 0.29496 & 0.29496 & 0.29496 & 0.29844 & 0.29496 \\
\hline
\end{tabular}


Table 2: Statistical analysis: Evaluation of WOAPO approach over existing approaches on IEEE 39 bus system

\begin{tabular}{llllll}
\hline Metrics & FF [13] & GA [12] & ABC [15] & PSO [14] & WOAPO \\
\hline Best & 36.298 & 36.298 & 36.298 & 36.28 & 36.192 \\
Mean & 36.303 & 36.301 & 36.359 & 36.413 & 36.286 \\
Standard deviation & 0.006306 & 0.003591 & 0.077518 & 0.09631 & 0.05332 \\
Median & 36.302 & 36.299 & 36.327 & 36.469 & 36.304 \\
Worst & 36.313 & 36.305 & 36.482 & 36.491 & 36.322 \\
\hline
\end{tabular}

\subsection{Analysis by Altering $\alpha$}

The WOAPO based approach accomplishes the minimal loss by changing the parameter $\alpha$ as specified by Eq. (3). The tabular manifestation attained by varying $\alpha$ using WOAPO model is shown in Table III for IEEE 14 bus system. Here, the value of $\alpha$ is varied and the losses, voltage penalties and final costs were computed. The loss is almost same for all $\alpha$ values. The VSI is minimum when selecting $\alpha=1$, and the reduced final fitness is attained when choosing $\alpha=4$. In Table IV, VSI is minimum when selecting $\alpha$ $=2$ and LSI is minimal when selecting $\alpha=3$ and the final cost is minimal when selecting $\alpha=0.1$.

Table 3: Analysis on ORPD by varying the values of a for IEEE 14 bus system

\begin{tabular}{llll}
\hline Varied values of $\alpha$ & LSI & VSI & Final cost \\
\hline$\alpha=1$ & 1 & 0.88 & 1 \\
$\alpha=2$ & 1 & 0.89 & 0.78 \\
$\alpha=3$ & 1 & 1 & 0.5 \\
$\alpha=4$ & 1 & 1 & 0.25 \\
\hline
\end{tabular}

Table 4: Analysis on ORPD by varying the values of $\alpha$ for IEEE 39 bus system

\begin{tabular}{llll}
\hline Varied values of $\alpha$ & LSI & VSI & Final cost \\
\hline$\alpha=1$ & 1 & 0.87 & 0.42 \\
$\alpha=2$ & 0.99 & 0.4 & 0.62 \\
$\alpha=3$ & 0.98 & 0.41 & 0.82 \\
$\alpha=4$ & 0.99 & 0.99 & 1 \\
\hline
\end{tabular}

\section{Conclusion}

This work has exploited WOAPO approach for resolving the issues in ORPD. In this work, the ORPD was portrayed as a non-linear issue and it was solved by evaluating the objectives namely minimisation of power loss and minimisation of variations in voltage. For attaining these objectives, WOAPO based optimization approach was deployed in this paper. Furthermore, the superiority of WOAPO approach was evaluated over other existing approaches and the outcomes were attained. On observing the outcomes, the values attained under best case scenario using WOAPO scheme was $0.57 \%, 0.5 \%$ and $0.92 \%$ improved than GA, FF and ABC. In addition, the worst case scenario of WOAPO approach was $1.16 \%$ superior to PSO model. Also, the values attained under mean case scenario using WOAPO technique was $0.12 \%, 0.12 \%, 0.78 \% \%$ better than $\mathrm{GA}, \mathrm{FF}$ and $\mathrm{ABC}$ models. Thus, the advantage of WOAPO approach for obtaining an enhanced ORPD model was validated effectively.

\section{Compliance with Ethical Standards}

Conflicts of interest: Authors declared that they have no conflict of interest.

Human participants: The conducted research follows the ethical standards and the authors ensured that they have not conducted any studies with human participants or animals.

\section{References}

[1] M. Ettappan, V. Vimala, S. Ramesh, V. Thiruppathy Kesavan, "Optimal reactive power dispatch for real power loss minimization and voltage stability enhancement using Artificial Bee Colony Algorithm”, Microprocessors and Microsystems, vol. 76, July 2020, Article 103085.

[2] Ali Asghar Heidari, Rahim Ali Abbaspour, Ahmad Rezaee Jordehi, "Gaussian bare-bones water cycle algorithm for optimal RP dispatch in electrical power systems”, Applied Soft Computing, vol. 57, pp. 657-671, August 2017. 
[3] K. Nuaekaew, Pramin Artrit, N. Pholdee, S. Bureerat, "Optimal RP dispatch problem using a two-archive multiobjective grey wolf optimizer”, Expert Systems with Applications, vol. 87, pp. 79-89, 30 November 2017.

[4] Rebecca Ng Shin Mei, Mohd Herwan Sulaiman, Zuriani Mustaffa, Hamdan Daniyal, "Optimal RP dispatch solution by loss minimization using moth-flame optimization technique", Applied Soft Computing, vol. 59, pp. 210-222, October 2017.

[5] Abhishek Rajan, T. Malakar, "Optimal RP dispatch using hybrid Nelder-Mead simplex based firefly algorithm", International Journal of Electrical Power \& Energy Systems, vol. 66, pp. 9-24, March 2015.

[6] Souhil Mouassa, Tarek Bouktir, Ahmed. Salhi, "Ant lion optimizer for solving optimal RP dispatch problem in power systems”, Engineering Science and Technology, vol. 20, no. 3, pp. 885-895, June 2017.

[7] Pradeep Jangir, Siddharth A. Parmar, Indrajit N. Trivedi, R. H. Bhesdadiya, "A novel hybrid Particle Swarm Optimizer with multi verse optimizer for global numerical optimization and Optimal RP Dispatch problem", Engineering Science and Technology, vol. 20, no. 2, pp. 570-586, April 2017.

[8] E Naderi, H. Narimani, Mehdi Fathi, M R Narimani, "A novel fuzzy adaptive configuration of particle swarm optimization to solve large-scale optimal RP dispatch", Applied Soft Computing, vol. 53, pp. 441-456, April 2017.

[9] Etta Grover-Silva, Robin Girard, George Kariniotakis, "Optimal sizing and placement of distribution grid connected battery systems through an SOCP optimal power flow algorithm”, Applied Energy, vol. 219, pp. 385393, 1 June 2018.

[10] Abdel-Fattah Attia, Ragab A. El Sehiemy, Hany M. Hasanien, "Optimal power flow solution in power systems using a novel Sine-Cosine algorithm", International Journal of Electrical Power \& Energy Systems, vol. 99, pp. 331-343, July 2018.

[11] Alexander Engelmann, Tillmann Mühlpfordt, Yuning Jiang, Boris Houska, Timm Faulwasser, "Distributed AC Optimal Power Flow using ALADIN", IFAC-Papers OnLine, vol. 50, no. 1, pp. 5536-5541, July 2017.

[12] Benjamin Rolf, Tobias Reggelin, Abdulrahman Nahhas, Sebastian Lang, Marcel Müller, "Assigning dispatching rules using a genetic algorithm to solve a hybrid flow shop scheduling problem" Procedia Manufacturing, vol. 42, pp. 442-449, 2020

[13] Hui Wang, Wenjun Wang, Xinyu Zhou, Hui Sun, Zhihua Cui, “Firefly algorithm with neighborhood attraction”, Information Sciences, pp. 374-387, vol. 382-383, March 2017.

[14] Junhao Zhang, Pinqi Xia, "An improved PSO algorithm for parameter identification of nonlinear dynamic hysteretic models", Journal of Sound and Vibration, vol. 389, pp. 153-167, 17 February 2017.

[15] K Z Gao, Ponnuthurai Suganthan, Quan, Mehmet, Ali Sadollah, "Artificial bee colony algorithm for scheduling and rescheduling fuzzy flexible job shop problem with new job insertion”, Knowledge-Based Systems, vol. 109, pp. 1-16, 1 October 2016.

[16] Sarath, K.S. \& Sekar, S, "Modelling and Optimal Design of LLC Resonant Converter using Whale Optimization Algorithm", International Journal of Modeling, 2018.

[17] Jian LiNi WangDao ZhouWeihao HuFrede Blaabjerg, "Optimal reactive power dispatch of permanent magnet synchronous generator-based wind farm considering levelised production cost minimisation”, Renewable Energy, vol.145, pp. 1-12, January 2020.

[18] Mohamed A. M. ShaheenHany M. HasanienAbdulaziz Alkuhayli, "A novel hybrid GWO-PSO optimization technique for optimal reactive power dispatch problem solution”, Ain Shams Engineering JournalIn press, corrected proofAvailable online 6 August 2020

[19] Partha P. BiswasP. N. SuganthanR. MallipeddiGehan A. J. Amaratunga, "Optimal reactive power dispatch with uncertainties in load demand and renewable energy sources adopting scenario-based approach", Applied Soft Computing, vol.75, pp. 616-632, February 2019. 